Polymer Journal, Vol. 9, No. 6, pp 605-611 (1977)

\title{
Small-Angle X-Ray Scattering of Poly(tetraoxane) Obtained by the Radiation-Induced Solid-State Polymerization
}

\author{
Yoshiaki NAKASE, ${ }^{*}$ Toshio Kato, ${ }^{*}$ Osamu YodA, ${ }^{*}$ Isamu KuriYAmA*, \\ and Akira ODAJIMA** \\ *Japan Atomic Energy Research Institute, Takasaki Radiation \\ Chemistry Research Establishment, Takasaki 370-12, Japan. \\ **Department of Applied Physics, Faculty of Engineering, \\ Hokkaido University, Sapporo 060, Japan.
}

(Received May 16, 1977)

\begin{abstract}
Intensities of small-angle X-ray scattering of a poly(tetraoxane) system, on both the direction of the equator and that of the meridian, increase by reducing the amount of unreacted tetraoxane, indicating the formation of voids.

A broad scattering maximum in the meridian besides a spot-like scattering corresponding to $100-\AA$ A spacing was observed in a polymer prepared at $105^{\circ} \mathrm{C}$. The broad maximum shifts to a low angle by annealing the sample below $140^{\circ} \mathrm{C}$, i.e., at a temperature range which has no influence on the polymer chain aggregation. The shift is associated with the sublimation of the unreacted monomer or the thermal decomposition of low-molecular-weight polymer. These materials are held in voids formed periodically on the fibrillar polymer crystal.

The fibrillar structure of poly(tetraoxane) seems to be an extended polymer chain aggregation with a periodic void appearance, i.e., a stacking of crystallites along the $c$-axis, while a lamellar crystal corresponding to 100 - $\AA$ spacing situates between these fibrils.
\end{abstract}

\section{KEY WORDS Poly(tetraoxane) / Small-Angle X-Ray Scattering / Annealing / Fibrillar Structure / Periodic Voids / Melting Behavior / Weight Loss /}

In a preceding paper, ${ }^{1}$ we reported that two discrete scatterings were observed in the meridional direction of small-angle $\mathrm{X}$-ray scattering (SAXS) patterns of poly(tetraoxane) ( $\mathrm{PT}_{\mathrm{E}} \mathrm{OX}$ ) postpolymerized at $105^{\circ} \mathrm{C}$, not showing twinned structures; one was a weak spot-like scattering corresponding to the Bragg spacing of about $100 \AA$ (100 $\AA$-scattering), and the other corresponding to about $500 \AA$ ( $500 \AA$-scattering). In the equatorial directions of a SAXS pattern, only continuous scattering was observed, indicating the existence of micro-voids between fibrils. ${ }^{2}$ It was clarified $^{1}$ that the $100 \AA$-scattering is due to lamellar crystallites having a thickness of $c a$. $100 \AA$, situated between fibrillar bundles of extended chain crystallites (main crystal).

The $500 \AA$-scattering reflects another fluctuation of an electron density along the fibrillar direction due to the extended chain crystallites.
In this paper, the origin of the $500 \AA$-scattering is investigated in detail by SAXS and the melting behavior of $\mathrm{PT}_{\mathrm{E}} \mathrm{OX}$.

\section{EXPERIMENTAL}

A single tetraoxane crystal prepared by sublimation under reduced pressure was post-polymerized at a certain temperature in the solid state. The polymer yield was determined gravimetrically after washing the sample with acetone and drying it under reduced pressure for $24 \mathrm{~h}$ at room temperature.

The annealing of $\mathrm{PT}_{\mathrm{E}} \mathrm{OX}$ was performed in a glass ampule at temperature below $140^{\circ} \mathrm{C}$. In annealing at temperatures below $140^{\circ} \mathrm{C}$, no increase in the long spacing of the $100 \AA$-scattering was observed nor was any effect of oxygen observed in either weight loss or melting behavior. ${ }^{1}$ 
The apparatus for the X-ray scattering measurements was the Rigaku Denki RU-3L rotating anode X-ray unit. The SAXS patterns were obtained photographically with a pin-hole collimation $(0.5 \mathrm{~mm} \phi$ and $0.3 \mathrm{~mm} \phi)$, and the SAXS intensity curves were recorded by a stepscan method with a Kratky U-slit $(20 \mu \mathrm{m}, 0.1$ $\mathrm{mm}$ and $0.05 \mathrm{~mm}$ ). The scattering intensity was uncertain at angle $(2 \theta)$ below $2^{\prime}$ because of the strong incident beam. The intensity at angles over $2^{\prime}$ has been corrected for air scattering.

The apparatus for measuring the melting behavior of $\mathrm{PT}_{\mathrm{E}} \mathrm{OX}$ was a differential scanning calorimeter (DSC-1B, Perkin-Elmer) with a small amount of the sample (ca. $1 \mathrm{mg}$ ). The measurements were performed at a heating rate of $16^{\circ} \mathrm{C}$ $\min ^{-1}$ under a nitrogen stream. The experimental details have been described elsewhere. ${ }^{1}$
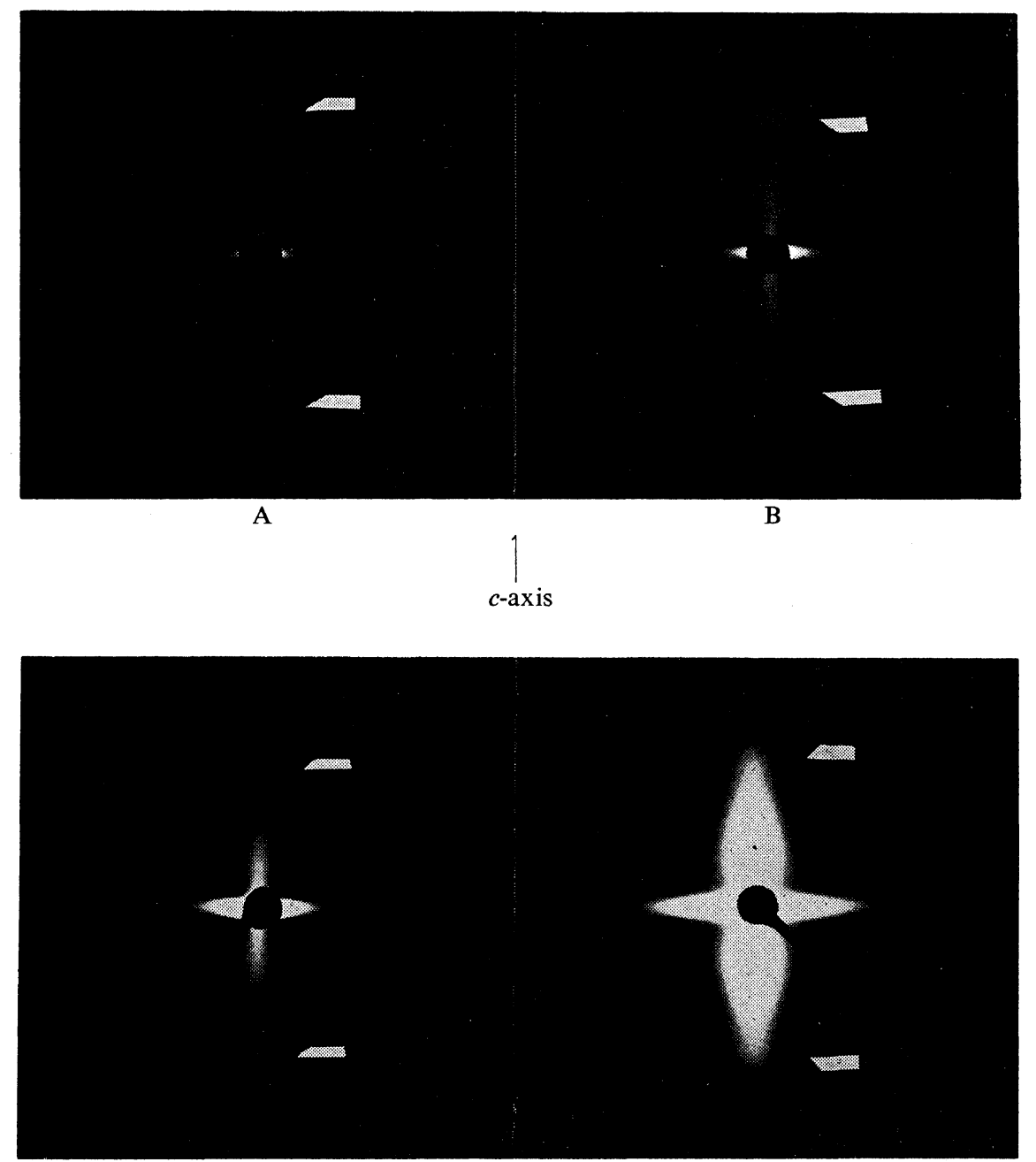

C

D

Figure 1. Small-angle $X$-ray scattering patterns of poly(tetraoxane) post-polymerized at $105^{\circ} \mathrm{C}$ for $15 \mathrm{~min}$ after the preirradiation of $0.1 \mathrm{MR}$ in air, polymer yield is $26 \%$ : (A), just after polymerization (before the extraction of unreacted monomers; (B), after the sublimation of unreacted monomer at $80^{\circ} \mathrm{C}$ for $3 \mathrm{~h}$; (C), after the sublimation for $5 \mathrm{~h}$; and (D), after the sublimation for $24 \mathrm{~h}$ (almost polymer alone). Half-arrows indicate spot-like scattering corresponding to $100 \AA$-spacing. 


\section{RESULTS AND DISCUSSION}

Figure 1 shows the SAXS patterns for various types of $\mathrm{PT}_{\mathrm{E}} \mathrm{OX}$ systems; (A), the system includes unreacted monomeric tetraoxane just after polymerization; (B), the system $\mathrm{A}$ is heated at $80^{\circ} \mathrm{C}$ for $3 \mathrm{~h}$ to sublime unreacted monomer; (C), the system $\mathrm{A}$ is heated for $5 \mathrm{~h}$; and (D), the system $\mathrm{A}$ is heated for $24 \mathrm{~h}$. The sample was washed with acetone and dried to give a polymer yield of $26 \%$. In the sample $A$, faint scatterings are observed in both directions of the equator and the meridian. A spot-like scattering in the meridian indicated by halfarrows is from the lamellar crystal with a Bragg spacing of $c a .100 \AA$ as mentioned already. ${ }^{1}$

The scattering intensities of both directions increase with the sublimation time $(B, C$, and $D)$, indicating an increase in the electron density fluctuation by a reduction of the residual monomer. The equatorial scattering was due to the needle-shaped voids between the fibrils, ${ }^{2}$ but the mechanism for the meridional scattering corresponding to the $500 \AA$-scattering has not been clarified yet. Therefore, the intensity curve at angles lower than $25^{\prime}$ in the meridional di-

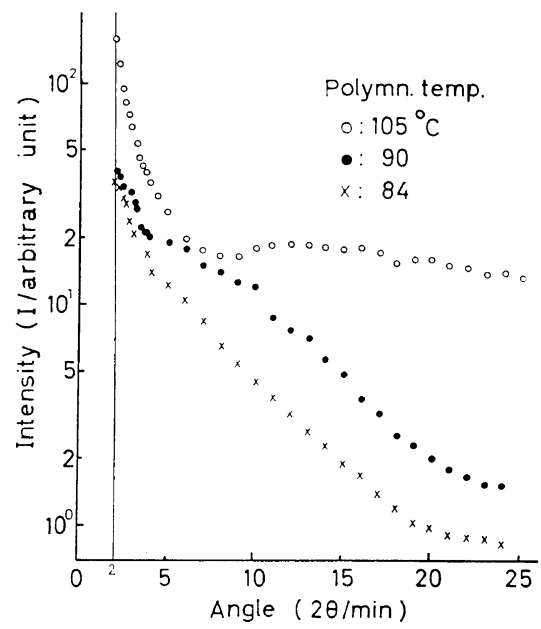

Figure 2. Small-angle X-ray scattering (meridian) curves of the sample prepared at several different temperatures: preirradiation, $1 \mathrm{MR}$; polymerization, for $1 \mathrm{~h}$ at $105^{\circ} \mathrm{C}$, for $14 \mathrm{~h}$ at $90^{\circ} \mathrm{C}$ and for $25 \mathrm{~h}$ at $84^{\circ} \mathrm{C}$ in air (ca. 30-\% yield in each case). Intensities are not comparable because of difference in sample size.

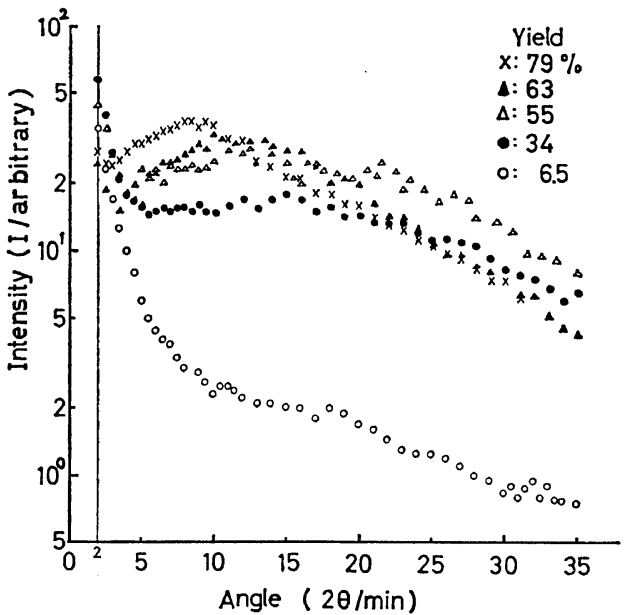

Figure 3. Small-angle X-ray scattering patterns of the samples with various polymer yields: polymerization at $105^{\circ} \mathrm{C}$ for a given time after the preirradiation of $1.0 \mathrm{MR}$ in air. Polymer yields are indicated in the figure.

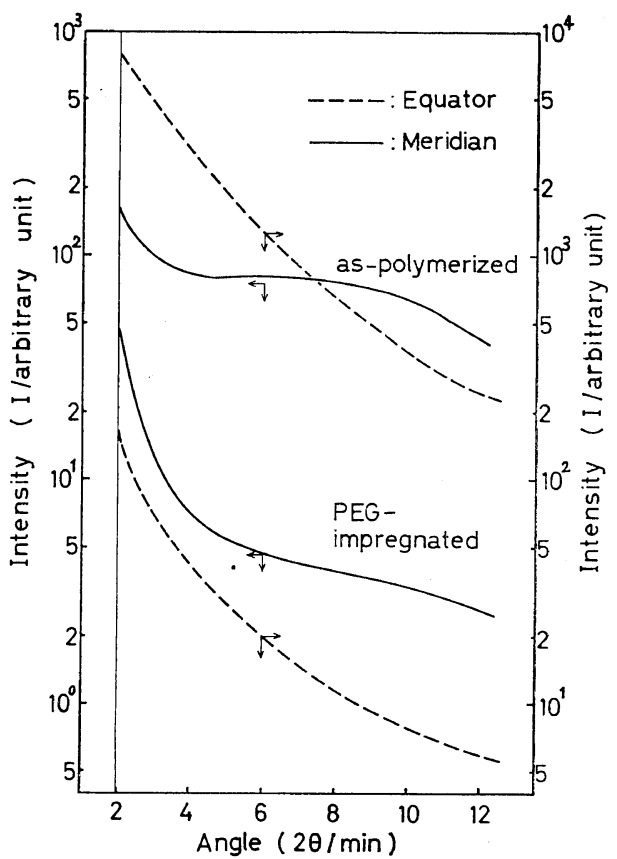

Figure 4. Effect of impregnation of poly(ethylene glycol) on the small angle X-ray scattering curves on equator and meridian of the sample polymerized at $105^{\circ} \mathrm{C}$ for $1 \mathrm{~h}$ (polymer yield, $40 \%$ ) after the preirradiation of $1 \mathrm{MR}$ in nitrogen atmosphere. 
rection was investigated using a Kratky U-slit camera.

Figure 2 shows the SAXS curves on the meridian of the $\mathrm{PT}_{\mathrm{E}} \mathrm{OX}$ obtained by the post-polymerization at various temperatures with a poly- mer yield of about $30 \%$ in each case. A broad scattering peak was observed at about $13^{\prime}$ (500 $\AA$ scattering) in the sample prepared at $105^{\circ} \mathrm{C}$, but a shoulder at about $10^{\prime}$ in the samples at $90^{\circ}$ and $84^{\circ} \mathrm{C}$. The intensity curve of $\mathrm{PT}_{\mathrm{E}} \mathrm{OX}$ pre-
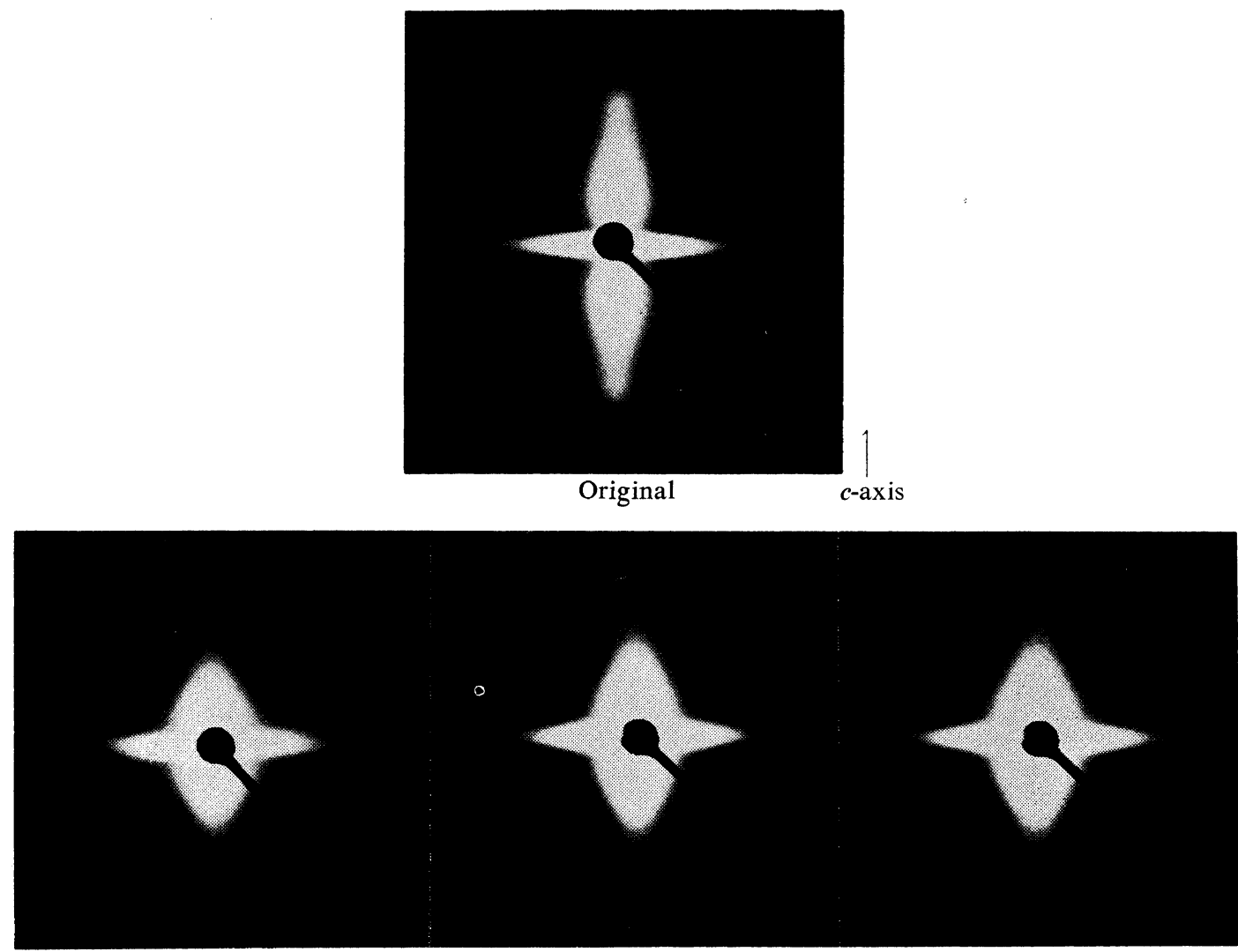

$75^{\circ} \mathrm{C}$

$100^{\circ} \mathrm{C}$

$110^{\circ} \mathrm{C}$

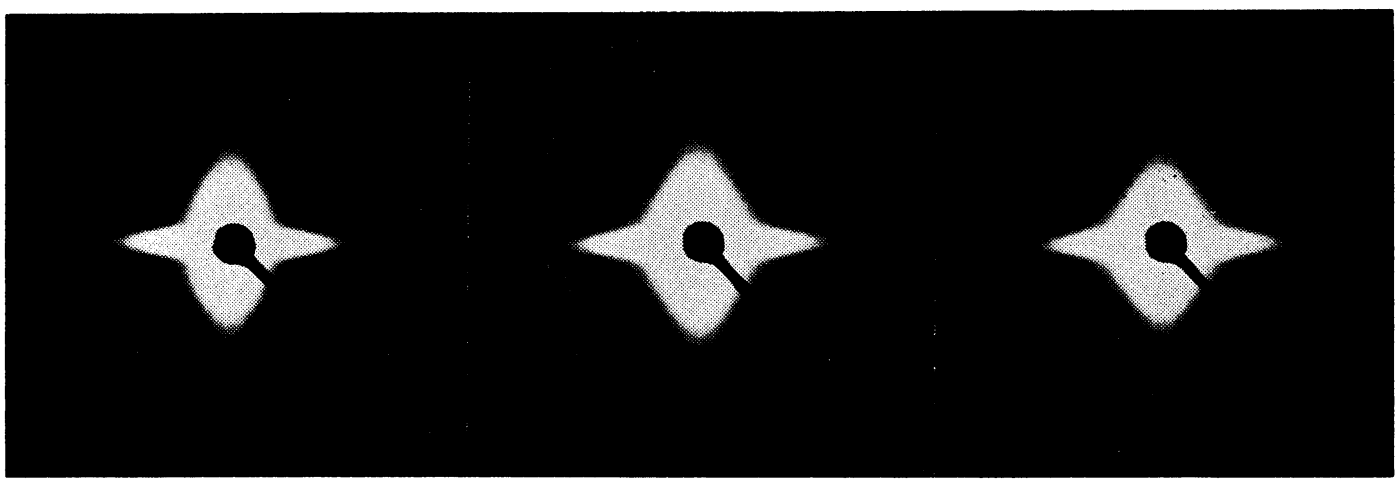

$120^{\circ} \mathrm{C}$

$130^{\circ} \mathrm{C}$

$140^{\circ} \mathrm{C}$

Figure 5. Small-angle X-ray scattering patterns of the sample annealed at various temperatures for $30 \mathrm{~min}$ except at $75^{\circ} \mathrm{C}$ for 8 days: polymerized at $105^{\circ} \mathrm{C} 1 \mathrm{~h}$ in air (yield $40 \%$ ) after $1 \mathrm{MR}$ irradiation. The annealing temperatures are indicated in the figure. 
pared at $70^{\circ} \mathrm{C}$ (polymer yield of about 10\%) is similar to that at $84^{\circ} \mathrm{C}$, indicating a rapid decrease in the intensity with the scattering angle.

The effect of the polymer yield on the broad peak (500 $\AA$-scattering) is examined in the sample prepared at $105^{\circ} \mathrm{C}$. Figure 3 shows the SAXS curves of $\mathrm{PT}_{\mathrm{E}} \mathrm{OX}$ with various polymer yields. Since the size and weight of the sample cannot be made equal for each sample, the intensities of SAXS cannot be compared. When the polymer yield is low, the scattering maximum at about $13^{\prime}$ or a higher angle can hardly be observed, and as the yield increases, the maximum becomes clearer and the peak position shifts to a lower angle size.

Figure 4 shows the reduction of the SAXS intensity by impregnation of poly(ethylene glycol) (PEG) in the directions of both the equator and the meridian. A similar result has been obtained with a sample impregnated with silicone oil. A great decrease in the SAXS intensity by impregnation suggests that the scatterings are caused by voids formed during the polymerization crystallization.

While investigating the annealing effect on spot-like scattering (100 $\AA$-scattering) in the meridional direction, it was found that an increase of the $100 \AA$-scattering occurred only at temperatures above $140^{\circ} \mathrm{C}$, but that the maximum of the broad $500 \AA$-scattering shifted to a lower angle by annealing a sample below $140^{\circ} \mathrm{C} .^{1}$

Figure 5 shows the SAXS patterns of $\mathrm{PT}_{\mathrm{E}} \mathrm{OX}$ (40-\% yield) as-polymerized, annealed for $30 \mathrm{~min}$ at various temperatures and annealed for 8 days at $75^{\circ} \mathrm{C}$. The annealing at $75^{\circ} \mathrm{C}$ for $100 \mathrm{~h}$ brought about no change in the SAXS patterns, but after $200 \mathrm{~h}$ a change was observed in the scattering patterns, as well as after $30 \mathrm{~min}$ in the temperature range between $100^{\circ} \mathrm{C}$ and $140^{\circ} \mathrm{C}$. On annealing $\mathrm{PT}_{\mathrm{E}} \mathrm{OX}$, a ring-like scattering pattern was noticed around the beam trap, suggesting the existence of unoriented voids.

The melting behaviors of the same samples as in Figure 5 are shown in Figure 6: The endothermic profile of the original $\mathrm{PT}_{\mathrm{E}} \mathrm{OX}$ changes so that the two peaks become a little clear by annealing, but, the total area under the double peak stays constant. This indicates that the aggregations of the polymer chains are affected little during annealing.

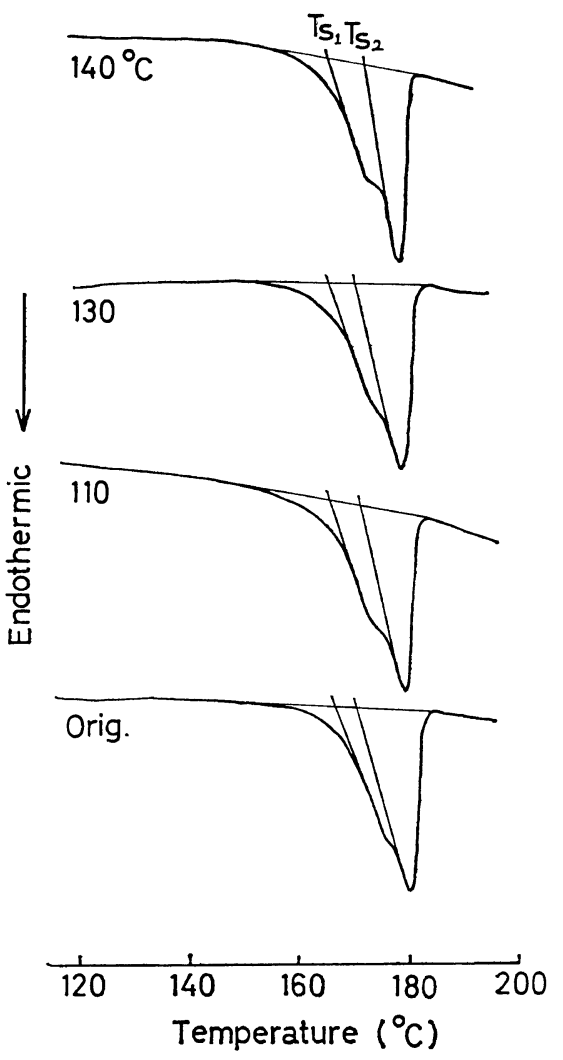

Figure 6. Heating curves of the samples annealed (the same sample used in Figure 5): heating rate, $16^{\circ} \mathrm{C} / \mathrm{min}$; sample weight used, $1.00 \pm 0.5 \mathrm{mg}$.

Table I. Weight loss during the annealing at various temperatures

\begin{tabular}{cc}
$\begin{array}{c}\text { Annealing } \\
\text { temperature, }{ }^{\circ} \mathrm{C}\end{array}$ & $\begin{array}{c}\text { Weight loss, } \\
\%\end{array}$ \\
\hline 75 & 5 \\
100 & 7 \\
110 & 10 \\
120 & 8 \\
130 & 10 \\
140 & 15
\end{tabular}

Table I summarizes the weight loss of the sample during annealing. The loss increases by raising the annealing temperature, and a maximum loss of $15 \%$ occurs in the sample annealed at $140^{\circ} \mathrm{C}$. If such an amount of weight loss is due to the thermal decomposition of high-molecular-weight polymers, the profiles of the melting endotherm as well as the scattering behavior 
must be influenced seriously. Thus, the weight loss during annealing is caused by the sublimation of unreacted monomers and/or by the decomposition of low-molecular-weight polymers.

The voids formed during polymerization retain unreacted monomers and/or low-molecularweight polymers, which are easily removed by annealing because of the high vapor pressure of the monomers and the thermal instability of low-molecular-weight polymers at elevated temperatures. These findings suggest us that the fibrillar crystal, or the extended chain crystal, must be composed of stacking crystallites.

Figure 7 shows the meridional SAXS intensity curves for $\mathrm{PT}_{\mathrm{E}} \mathrm{OX}$ (the same sample used in Figure 5). The original sample shows a very broad intensity maximum at about $13^{\prime}$, the position of which shifts to a low angle side by annealing the sample. This indicates an increase in the spacing of the void appearance by annealing $\mathrm{PT}_{\mathrm{E}} \mathrm{OX}$.

Figure 8 shows the SAXS curves of $\mathrm{PT}_{\mathrm{E}} \mathrm{OX}$ obtained at $105^{\circ} \mathrm{C}$ in vacuum. The profile of the SAXS intensity curve of the original changes similarly to that of the sample post-polymerized in air (Figure 7) during annealing, indicating no special effect of oxygen on the $500 \AA$ scattering. The length of the crystallites men-

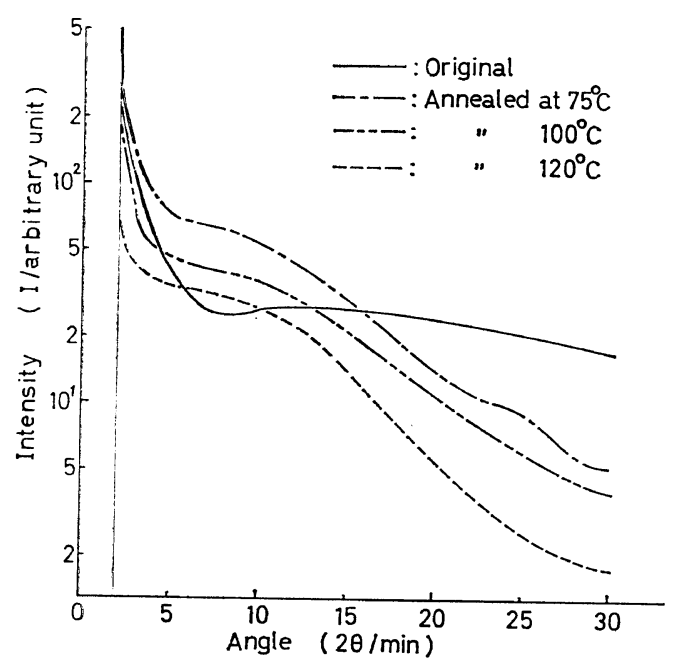

Figure 7. Small-angle X-ray scattering curves of the sample annealed (the sample used in Figure 5). The annealing temperatures are indicated in the figure.

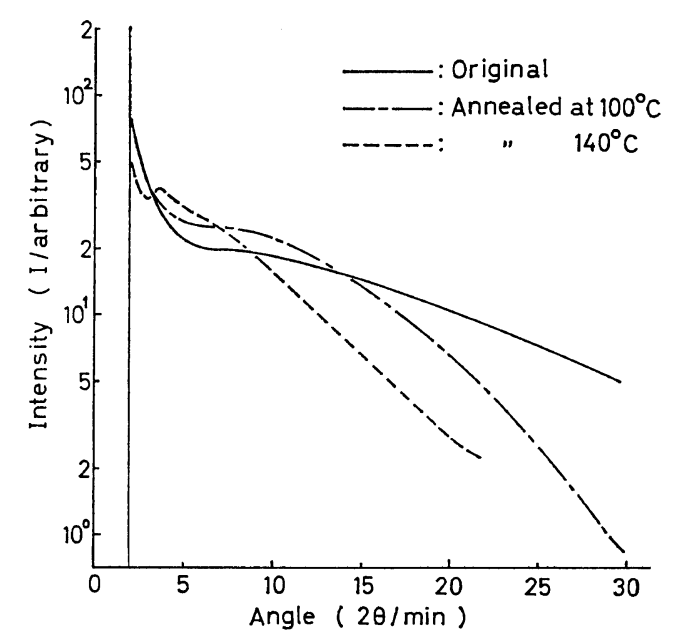

Figure 8. Small-angle X-ray scattering curves of the sample prepared in vacuum: polymerization at $105^{\circ} \mathrm{C}$ for $1 \mathrm{~h}$ after $1 \mathrm{MR}$ preirradiation in vacuum (polymer yield, 36\%).

tioned above, therefore, does not reflect the molecular weight of the polymers, since the post-polymerization of $\mathrm{PT}_{\mathrm{E}} \mathrm{OX}$ in air gives a polymer with a lower molecular weight than that in vacuum ${ }^{3}$. The periodic appearance of voids on the polymer fibrils prepared both in air and in vacuum is a characteristic of the $\mathrm{PT}_{\mathrm{E}} \mathrm{OX}$ texture, having to do mainly with the difference in unit cell dimensions ${ }^{4}$ of both the crystals of monomeric $\mathrm{T}_{\mathrm{E}} \mathrm{OX}$ and $\mathrm{PT}_{\mathrm{E}} \mathrm{OX}$.

\section{CONCLUSIONS}

The texture of $\mathrm{PT}_{\mathrm{E}} \mathrm{OX}$ post-polymerized at a temperature below $80^{\circ} \mathrm{C}$ is different from that of $\mathrm{PT}_{\mathrm{E}} \mathrm{OX}$ prepared above $80^{\circ} \mathrm{C}$. The SAXS patterns of the sample obtained at $105^{\circ} \mathrm{C}$, which has no twinned structure, have a broad maximum besides a spot-like scattering due to a lamellar crystal with a ca. $100-\AA$ spacing $^{1}$ on the meridian, and a diffuse scattering on the equator. Both scatterings are caused by voids; the former broad maximum is the scattering from the voids arranged periodically on a fibrillar crystal and the latter from those between the fibrils.

The voids are filled with unreacted monomers and/or low-molecular-weight polymers in the polymerization, and the monomers in the needle- 


\section{SAXS of $\mathrm{PT}_{\mathrm{E}} \mathrm{OX}$}

shaped voids between fibrils can be easily washed off with acetone, but materials situated between the crystallite ends in the fibrils are occluded rather tightly and are hard to wash off with acetone. The broad SAXS intensity maximum in the meridional direction is affected by the materials in the voids. The annealing reduces these materials by the sublimaiton of unreacted monomers and/or by the thermal decomposition of low-molecular-weight polymers to emphasize the periodic appearance of voids.

The characteristics of the voids in $\mathrm{PT}_{\mathrm{E}} \mathrm{OX}$ must be related to the polymerization mechanism in the solid state, and this can be clarified by quantitative analysis of X-ray diffraction behavior.

\section{REFERENCES}

1. T. Kato, Y. Nakase, O. Yoda, I. Kuriyama, and A. Odajima, Polym. J., 8331 (1976).

2. W. O. Statton, J. Polym. Sci., 58205 (1962).

3. Y. Nakase, M. Yoshida, A. Ito, and K. Hayashi, J. Polym. Sci., Part A-1, 9465 (1971); ibid., 10, 2181 (1972).

4. Y. Chatani, T. Uchida, H. Tadokoro, K. Hayashi, M. Nishii, and S. Okamura, J. Macromol. Sci.-Phys., B2, 567 (1968). 